\title{
Caractérisation Des Propriétés Hydrodynamiques Des Aquifères Du Socle Dans Quelques Localités Du Nord De La Côte d'Ivoire
}

\author{
Omer Zephir De Lasme \\ Stéphane Delmas Kouadio \\ Université Péléforo Gon Coulibaly, Korhogo-Côte d'Ivoire \\ Adama Coulibaly \\ Université Félix Houphouët-Boigny, Côte d'Ivoire
}

Doi:10.19044/esj.2021.v17n7p137

Submitted: 16 November 2020

Accepted: 11 January 2021

Published: 28 February 2021
Copyright 2021 Author(s)

Under Creative Commons BY-NC-ND

4.0 OPEN ACCESS

Cite As:

Zephir De Lasme O., Delmas Kouadio S. \& Coulibaly A. (2021). Caractérisation Des Propriétés Hydrodynamiques Des Aquifères Du Socle Dans Quelques Localités Du Nord De La Côte d'Ivoire. European Scientific Journal, ESJ, 17(7), 137.

https://doi.org/10.19044/esj.2021.v17n7p137

\section{Résumé}

Cette étude est focalisée sur la caractérisation des propriétés hydrodynamiques des aquifères du socle dans six localités du nord de la Côte d'Ivoire : Ganaoni, M'bengue, Tongon, Nielle, Ferke et Tafire. L'objectif principal est d'évaluer la productivité des aquifères de ces localités. La base de données est essentiellement constituée par les fiches techniques de 10 forages contenant les résultats des essais de pompage et le rapport de foration. L'approche méthodologique débute par le calcul de la valeur de la transmissivité selon la méthode de Cooper-Jacob, puis le débit spécifique par le quotient du débit pompé et le rabattement mesuré, et enfin déterminer le débit critique à l'aide de l'Outil d'Aide à l'Interprétation des Pompages (OUAIP) conçu par des chercheurs du Bureau de Recherches Géologiques et Minières de la France. Les données acquises ont fait l'objet d'analyses statistiques descriptives et multivariées pour établir les équations de corrélation prédictive entre les paramètres hydrodynamiques des aquifères. La transmissivité est comprise entre 4,01.10-6 m2.s-1 et 1,18.10-4 m2s-1 pendant que le débit spécifique varie entre 5,04.10-2 et 4,38.10-1 $\mathrm{m} 2 \mathrm{~h}-1$. Le débit critique est compris entre 1,05 et $6 \mathrm{~m} 3 / \mathrm{h}$. Les corrélations entre le débit 
critique (Qc) et le débit d'exploitation $(\mathrm{Q})$ puis, entre la transmissivité $(\mathrm{T})$ et le débit spécifique (Qsp) sont respectivement traduites par les équations prédictives : Qc $(\mathrm{m} 3 \mathrm{~h}-1)=0.63+0.61 \mathrm{Q}$ et $\mathrm{T}(\mathrm{m} 2 . \mathrm{h}-1)=0.63 \mathrm{Qsp} 1.3$. La productivité dépend du potentiel hydrodynamique intrinsèque des aquifères et le calibrage des ouvrages hydrauliques de prélèvement. Ces résultats permettent d'envisager une meilleure gestion des ressources en eaux souterraines à travers la modélisation des écoulements et l'usage des modèles géostatistiques.

Mots clés : Essais De Pompage, Hydrodynamique, Transmissivité, DébitSpécifique, Débit-Critique

\title{
Hydrodynamic Properties Characterization of Basement Aquifers in Few Localities in the North of Côte d'Ivoire
}

\author{
Omer Zephir De Lasme \\ Stéphane Delmas Kouadio \\ Université Péléforo Gon Coulibaly, Korhogo-Côte d'Ivoire \\ Adama Coulibaly \\ Université Félix Houphouët-Boigny, Côte d'Ivoire
}

\section{Abstract}

This study focused on the characterization of hydrodynamic properties of basement aquifers in six localities in the North of Côte d'Ivoire: Ganaoni, M'bengue, Tongon, Nielle, Ferke, and Tafire. The main objective is to assess the productivity of aquifers of these localities. The database is essentially pumping tests and drilling reports of 10 boreholes with the geological map. The methodological approach begins by calculating the value of transmissivity according to the Cooper-Jacob method, then the specific yield by the ratio of pumped flow rate and the measured drawdown, and finally determine the critical yield using the Pumping Interpretation Assistance Tool (OUAIP) designed by researchers from the Geological and Mining Research Office of France. The data acquired were subjected to descriptive and multivariate statistical analyzes to establish predictive correlation equations between hydrodynamic parameters of aquifers. Critical yield (Qc) of borehole evolved between 1.05 and $6 \mathrm{~m} 3 \mathrm{~h}-1$, while the specific yield (Qsp) ranged from 5.04.102 to $4.38 .10-1 \mathrm{~m} 2 \mathrm{~h}-1$, and transmissivity varied from $4.01 .10-6 \mathrm{~m} 2 \mathrm{~s}-1$ to 1.18.10-4 m2s-1. Correlated parameters allowed to write predictive equations below: Qc $(\mathrm{m} 3 \mathrm{~h}-1)=0,63+0,61 \mathrm{Q}(\mathrm{m} 3 . \mathrm{h}-1)$ and $\mathrm{T}(\mathrm{m} 2 \mathrm{~h}-1)=0,63 \mathrm{Qsp} 1,3$ (m2.h-1). Productivity depends on intrinsic hydrodynamical potentialities of aquifers and the calibration of pumping wells. Based on these results, the 
better management of groundwater resources can improve through flow modeling and geostatistical patterns using.

Keywords: Pumping-Test, Hydrodynamic, Transmissivity, Specific Yield, Critical Yield

\section{Introduction}

La pénurie d'eau est devenue un problème crucial vécu par toutes les sociétés et particulièrement celles des pays en voie de développement dont la Côte d'Ivoire qui, à travers son gouvernement et ses partenaires au développement, a initié un vaste programme national d'hydraulique villageoise à partir de 1973 (Lasm et al., 2012). Cependant, la disponibilité de l'eau potable pour la couverture totale des besoins alimentaires des populations fut une équation difficile à résoudre pendant le premier semestre de l'année 2018 dans la zone centre-nord de la Côte d'Ivoire notamment à Bouaké, Niakara, Ferké, Doropo, Odienné, et Tiéningboué, (Konan, 2018). En effet, la plupart des forages équipés de pompes à motricité humaine (système hydraulique villageoise) implantés pour le captage des eaux souterraines ne sont plus en état d'usage dans ces régions. Certaines des raisons de l'abandon de ces ouvrages hydrauliques sont les pannes récurrentes des pompes, et l'insuffisance des débits délivrés pendant l'exploitation occasionnant les nombreuses heures et files d'attente. Ainsi, chercher à connaître les propriétés hydrodynamiques intrinsèques des réservoirs et les limites d'exploitation des ouvrages hydrauliques implantés dans ces zones revêt une grande importance dans le cadre de la gestion durable des ressources d'eaux souterraines. C'est dans cette optique que cette étude a été menée avec le thème suivant : caractérisation des propriétés hydrodynamiques des aquifères du socle dans quelques localités du Nord de la Côte d'Ivoire.

\section{Situation Geographique, Contexte Geologique Et Hydrogeologique De La Zone D'etude}

La présente étude concerne les forages isolés dans les localités des régions suivantes (figure 1): Bagoué (Ganaoni), Tchologo (Niellé, Tongon, Diawala et Ferké), Poro (Sékonkaha) et le Hambol (Tafiré). 


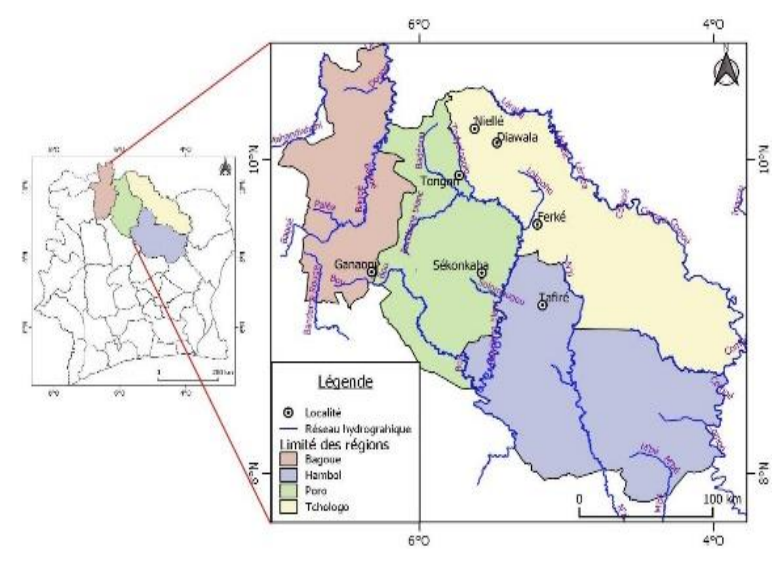

Figure 1. Localisation de la zone d'étude et des forages étudiés

Ces localités se trouvent dans le domaine du socle cristallin et cristallophyllien de la Côte d'Ivoire où les formations dominantes sont métamorphiques et magmatiques avec des reliques volcano-sédimentaires (voir figure 2). La géomorphologie se caractérise par un vaste plateau dont l'altitude varie entre 300 et $600 \mathrm{~m}$ (Geomines, 1982) avec une monotonie du relief interrompue par quelques rares collines dont la plus importante, Ouamelekoro (587 m) se trouve au Nord de Niellé (Kouakou et al., (2012). Sur le plan hydrologique, les localités concernées sont situées sur deux bassins versants principaux : Bandama et Volta Noire. Le bassin versant du Bandama a une superficie de $97000 \mathrm{Km}^{2}$. Il englobe les localités de Ganaoni, M'bengué, Tafiré et Tongon. Le bassin versant de la Volta Noire a une superficie de 82 $516 \mathrm{Km}^{2}$. Dans ce bassin, seule la localité de Niellé s'y trouve. Le réseau hydrographique de ces deux bassins est plus ou moins dense, avec les affluents du Bandama (Solomougou, Bou, Badenou) et de N'Zi (Lomi). Le Lomi passe plus au Nord de l'agglomération de Tafiré (Atté, 2010). Les récentes études sur les modèles conceptuels des aquifères du socle ont mis en évidence 3 différents types d'aquifères superposés en stratiforme ou non : les altérites, les horizons fissurés et les fractures profondes. Ces aquifères possèdent des propriétés hydrodynamiques singulières variables dans l'espace selon l'hétérogénéité du milieu de socle (Lachassagne et al., 2014). La productivité des réservoirs fracturés reste tributaire de la géométrie de la fracturation (Lasm, 2000). Les études hydrogéologiques précédentes en Côte d'ivoire s'accordent à identifier dans plusieurs localités une productivité supérieure des aquifères de schistes devant les granites (Biémi, 1992) sous des épaisseurs d'altération plus ou moins importantes n'excédant pas la trentaine de profondeur (Soro, 2010 ; De Lasme, 2013). 


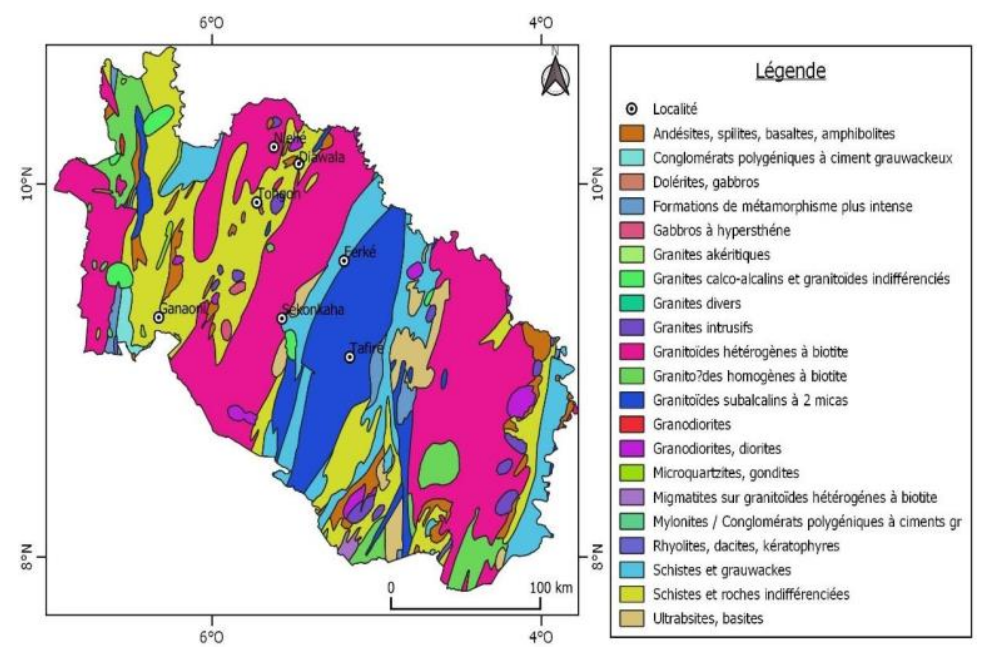

Figure 2. Carte géologique de la zone d'étude

\section{Matériel Et Méthodes Matériel}

Le matériel ayant servi à réaliser cette étude est constitué des fiches techniques de forages d'eau. Ces fiches sont les résultats des essais de pompage et les procès-verbaux (rapports écrits) de foration et équipement d'ouvrages hydrauliques. Ces fiches ont été collectées à la direction technique de l'hydraulique du département de Korhogo. Le traitement de ces données brutes a nécessité le recours à la carte géologique du Nord de la Côte d'Ivoire et de certains logiciels spécifiques :

- STATISTICA version 7.1 a permis d'effectuer des analyses statistiques,

- OUAIP version 1.3 a été utile pour déterminer de manière automatique le débit critique exploitable dans les aquifères, et le paramètre hydrodynamique lorsque cela était possible dans les conditions théoriques satisfaites. Face à certaines exigences non satisfaites, le logiciel AQTESOLV a aussi été utilisé pour la même cause, à savoir le calcul des valeurs des paramètres hydrodynamiques.

- QGIS Desktop 3.12.2 a été utilisé pour la localisation des forages sur la carte géologique du Nord de la Côte d'Ivoire.

\section{Methodes}

\section{Détermination Des Valeurs Des Paramètres Hydrodynamiques}

Le débit critique est le rendement à ne pas dépasser pendant l'exploitation au risque d'accélérer la détérioration de l'ouvrage et la mauvaise qualité de l'eau prélevée. La valeur du débit critique est déterminable graphiquement en identifiant sur la courbe caractéristique le point d'équivalence entre les pertes de charges linéaires et quadratiques lors des 
essais de pompages de courte durée. Le logiciel OUAIP calcule automatiquement la valeur du débit à ce point d'équivalence.

Le débit spécifique noté $\mathrm{Qsp}\left(\mathrm{m}^{2} / \mathrm{h}\right)$ est le paramètre traduisant la productivité intrinsèque de l'aquifère. Il est calculé par le quotient du débit pompé et du rabattement mesuré (équation 1 ) :

$$
\begin{aligned}
& Q_{s p}=\frac{Q}{s} \\
& -\quad \text { Q : débit de pompage }\left(\mathrm{m}^{3} / \mathrm{h}\right) \\
& -\quad \text { s: rabattement induit par le pompage (m) }
\end{aligned}
$$

Le débit considéré dans cette étude est celui du premier palier (premier cycle logarithmique) qui induit naturellement un rabattement du niveau de la nappe pendant la durée du pompage. Le choix du premier palier est dû au fait qu'il a mis plus de temps ( 2 heures) alors que les autres paliers (deuxième et troisième paliers) se déroulent généralement en moins de temps que le premier (Ehoussou et al., 2018).

-La transmissivité des aquifères est déterminable graphiquement à travers l'approximation logarithmique proposée par Cooper-Jacob basée sur l'équation de Theis (équation 2). Le logiciel OUAIP propose l'interprétation des résultats d'essais de pompages suivant plusieurs solutions analytiques dont celle de Cooper-Jacob qui a été choisie en raison de la quasi-satisfaction des conditions d'application de cette méthode employée par plusieurs auteurs ayant travaillé dans les zones de socle en Côte d'Ivoire (Biémi, 1992 ; Soro et al., 2010 ; Kouassi, 2011 ; De Lasme, 2012).

$$
s=\frac{Q}{4 \pi T} \ln \left(\frac{2.25 T t}{r^{2} S}\right)
$$

Avec :

$\mathrm{T}:$ transmissivité $\left(\mathrm{m}^{2} / \mathrm{s}\right)$

$\mathrm{s}$ : rabattement exprimé en $\mathrm{m}$;

$\mathrm{S}$ : coefficient d'emmagasinement, sans dimension;

$\mathrm{t}$ : temps écoulé depuis le début du pompage, exprimé en $\mathrm{s}$;

$\mathrm{r}$ : distance du piézomètre à l'axe du puits, en $\mathrm{m}$.

L'approximation logarithmique s'appuie sur des hypothèses de simplification et, elle permet de calculer la valeur de la transmissivité par l'équation 3 suivante :

$T=\frac{0,183 Q}{\Delta s}$

eq.

Avec:

$\mathrm{T}$ : transmissivité exprimée en $\mathrm{m}^{2} / \mathrm{s}$;

$\mathrm{Q}$ : débit de pompage du premier palier en $\mathrm{m}^{3} / \mathrm{s}$;

$\Delta \mathrm{s}$ : variation du rabattement $(\mathrm{m})$ sur un cycle logarithmique du temps.

Les récents travaux de recherche en hydrogéologie quantitative du bureau de recherche géologique et minière en France recommandent désormais l'application préalable de la méthode de diagnostic en vue de réduire les incertitudes des résultats issus des solutions analytiques (Gutierrez et Dewandel, 2013). Le diagnostic consiste en l'analyse de la dérivée 
logarithmique des rabattements (ds /dlnt) à la descente ou à la remontée, ce qui a l'avantage de représenter tous les régimes d'écoulement sur un seul et même graphique bi-logarithmique (Bourdet et al., 1983). Dans ce travail, la phase de diagnostic par l'analyse de la dérivée a été prise en compte car les résultats du calcul de la transmissivité ont été obtenus sous la version essai du logiciel AQTESOLV (Aquifer Test Solver) développé par la compagnie Hydrosolve aux Etats Unis d'Amérique. L'usage de ce logiciel a l'avantage d'afficher les graphiques du diagnostic puis d'effectuer automatiquement le calcul avec la solution analytique la plus idoine (Duffield, 2007)

\section{Classification Des Paramètres Hydrogéologiques}

Les paramètres hydrodynamiques tels que le débit spécifique et la transmissivité ont été classés en tenant compte des classes définis par des chercheurs dans des études antérieures similaires. Ainsi, pour mieux apprécier la distribution des débits de forage, le Comité Interafricain d'Études Hydrauliques (C.I.E.H.) a proposé les classifications suivantes (Lasm, 2000) :

- Classification des débits spécifiques

Qsp $<0,1 \mathrm{~m}^{2} / \mathrm{h}$ : débit spécifique faible ;

$0,1 \mathrm{~m}^{2} / \mathrm{h}<\mathrm{Q}_{\mathrm{sp}}<1 \mathrm{~m}^{2} / \mathrm{h}$ : débit spécifique moyen ;

Qsp $>1 \mathrm{~m}^{2} / \mathrm{h}$ : débit spécifique fort.

- Classification de la transmissivité

Classe faible : $\mathrm{T}<10^{-5} \mathrm{~m}^{2} / \mathrm{s}$;

Classe moyenne : $10^{-5} \mathrm{~m}^{2} / \mathrm{s}<\mathrm{T}<10^{-4} \mathrm{~m}^{2} / \mathrm{s}$;

Classe forte : $\mathrm{T}>10^{-4} \mathrm{~m}^{2} / \mathrm{s}$.

\section{Analyse De Corrélation Des Paramètres}

Afin de connaître l'influence des paramètres hydrodynamiques sur la productivité des forages, il a été effectué deux approches d'analyses statistiques. La première approche statistique consiste à établir des cercles de communautés afin d'identifier lesquels des paramètres évalués exercent une influence significative sur la productivité des aquifères. Cette approche a été réalisée à l'aide du logiciel STATISTICA suivant une analyse en composantes principales normées (ACPN) avec six (6) variables : la profondeur totale $(\mathrm{Pt})$, la transmissivité (T), le niveau statique (NS), le débit d'exploitation (Q), le débit critique $\left(\mathrm{Q}_{\mathrm{c}}\right)$ et le débit spécifique $\left(\mathrm{Q}_{\mathrm{sp}}\right)$. La seconde approche permet de mettre en relation empirique deux à deux, les différents paramètres susceptibles d'être fortement corrélés tels que Pt (Profondeur totale) et $\mathrm{Q}$ (Débit d'exploitation); Q (Débit d'exploitation) et Qc (Débit critique) puis $\mathrm{Q}_{\text {sp }}$ (Débit Spécifique) et $\mathrm{T}$ (Transmissivité). Cette seconde approche statistique a consisté à concevoir un nuage de points puis d'écrire des équations de droites de régression. 


\section{Resultats Et Discussion \\ Resultats \\ Analyses Statistiques}

Les valeurs des paramètres hydrauliques des ouvrages de captage et celles des aquifères sont consignées dans le tableau I qui présente les principales caractéristiques statistiques descriptives.

Tableau I. Statistiques descriptives des paramètres hydrodynamiques

\begin{tabular}{|l|l|l|l|l|l|l|}
\hline & $P t(m)$ & $N S(m)$ & $Q\left(m^{3} \cdot h^{-1}\right)$ & $Q_{c}\left(m^{3} \cdot h^{-}\right)$ & $Q_{s p}\left(m^{2} \cdot h^{-1}\right)$ & $T\left(m^{3} \cdot s^{-1}\right)$ \\
\hline Effectif & 10 & 10 & 10 & 10 & 10 & 10 \\
\hline Moyenne & $\mathbf{6 5 , 4 1}$ & 7,14 & 3,77 & 2,98 & $2,02.10^{-1}$ & $2,75.10^{-5}$ \\
\hline Variance & $\mathbf{1 1 4 3 , 4 2}$ & 20,71 & 4,61 & 1,97 & $\mathbf{0 , 0 1}$ & $\mathbf{0 , 0 1}$ \\
\hline Écart-type & $\mathbf{3 3 , 8 1}$ & 4,55 & 2,15 & 1,40 & $1,19.10^{-1}$ & $3,41.10^{-5}$ \\
\hline Minimum & 31,00 & 1,30 & 0,85 & 1,05 & $5,04 \cdot 10^{-2}$ & $4,01.10^{-6}$ \\
\hline Maximum & 119,86 & 18,15 & 8,00 & 6 & $4,38.10^{-1}$ & $1,18.10^{-4}$ \\
\hline CV $(\%)$ & 51,70 & 63,73 & 57,07 & $\mathbf{4 8 , 1 4}$ & 59.03 & 123.96 \\
\hline
\end{tabular}

L'effectif des échantillons est faible mais non négligeable dans un contexte de milieu discontinu où l'homogénéité spatiale des propriétés hydrodynamiques ne peut être satisfaite totalement. La variance et l'écart-type de la profondeur forée sont assez éloignés de la valeur moyenne mais ils sont quasiment dans le même ordre de grandeur pour la transmissivité et le débit spécifique. De même, la variance et l'écart-type du débit critique et du débit d'exploitation ne sont pas assez éloignés de la moyenne. Cependant, le coefficient de variation de la transmissivité est extrêmement élevé, ce qui indique son état de dispersion autour de la moyenne.

\section{Classification Des Paramètres}

La fréquence de distribution des valeurs de débits spécifiques selon la classification proposée par le CIEH est présentée par la figure 3 . Elle révèle que $80 \%$ des débits spécifiques sont moyens et $20 \%$ d'entre eux sont classés faibles.

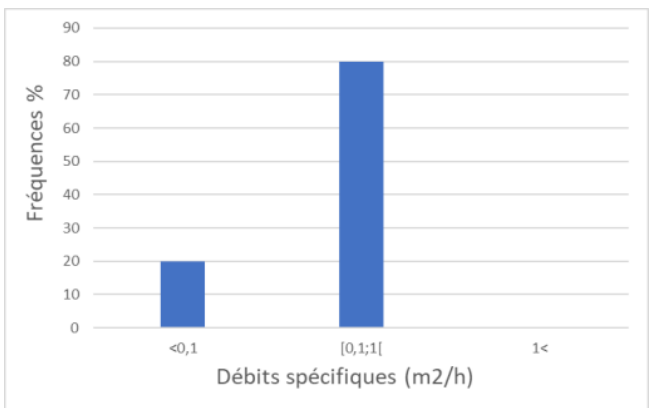

Figure 3. Fréquence de distribution des valeurs de débits spécifiques 
La fréquence de distribution des valeurs de débits spécifiques selon la classification proposée par le CIEH est présentée par la figure 4. Elle montre que seulement $10 \%$ des valeurs de la transmissivité sont dans la classe forte ; pendant que la classe moyenne et la classe faible totalisent respectivement $60 \%$ et $30 \%$ de l'effectif total étudié.

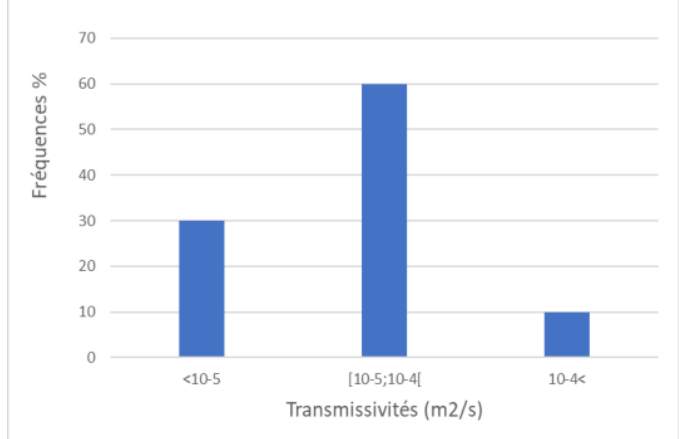

Figure 4. Fréquence de distribution des valeurs de la transmissivité

$\mathrm{Au}$ regard de cette classification, les aquifères étudiés peuvent être communément caractérisés par une transmissivité moyenne et un débit spécifique moyen.

\section{Correlation Entre Les Parametres}

L'analyse en composantes principales normées a mis en évidence, à travers la matrice de corrélation, le niveau de corrélation qui existe entre les différents paramètres évalués (tableau III) :

Tableau III. Matrice de corrélation des paramètres

\begin{tabular}{|l|l|l|l|l|l|l|}
\hline & $P t(m)$ & $N S(m)$ & $Q\left(\mathrm{~m}^{3} / \mathrm{h}\right)$ & $Q_{s p}\left(\mathrm{~m}^{2} / \mathrm{h}\right)$ & $T\left(\mathrm{~m}^{2} / \mathrm{s}\right)$ & $\begin{array}{c}Q_{c} \\
\left(\mathrm{~m}^{3} / \mathrm{h}\right)\end{array}$ \\
\hline $\mathrm{Pt}(\mathrm{m})$ & 1,00 & & & & & \\
\hline $\mathrm{NS}(\mathrm{m})$ & $\mathbf{- 0 , 6 4}$ & 1,00 & & & & \\
\hline $\mathrm{Q}\left(\mathrm{m}^{3} / \mathrm{h}\right)$ & $\mathbf{0 , 6 2}$ & $-0,38$ & 1,00 & & & \\
\hline $\mathrm{Q}_{\mathrm{sp}}\left(\mathrm{m}^{2} / \mathrm{h}\right)$ & $-0,02$ & 0,13 & $\mathbf{0 , 5 6}$ & 1,00 & & \\
\hline $\mathrm{T}\left(\mathrm{m}^{2} / \mathrm{s}\right)$ & $-0,34$ & 0,24 & 0,16 & $\mathbf{0 , 8 5}$ & 1,00 & \\
\hline $\mathrm{Q}_{\mathrm{c}}\left(\mathrm{m}^{3} / \mathrm{h}\right)$ & $\mathbf{0 . 6 3}$ & -0.48 & $\mathbf{0 , 9 3}$ & 0,33 & $-0,13$ & 1,00 \\
\hline
\end{tabular}

Il se dégage les corrélations pertinentes suivantes :

$\checkmark$ très bonne corrélation entre $\mathrm{T}$ et $\mathrm{Q}_{\mathrm{sp}}(0,85)$ puis entre $\mathrm{Q}$ et $\mathrm{Q}_{\mathrm{c}}(0,93)$

$\checkmark$ moyenne corrélation de $\mathrm{Pt}$ avec $\mathrm{Q}(0,62), \mathrm{Pt}$ avec $\mathrm{Q}_{\mathrm{c}}(0,63)$ et $\mathrm{Q}$ avec $\mathrm{Q}_{\mathrm{sp}}(0,56)$

$\checkmark$ très faible corrélation $(0,16)$ de la transmissivité $(\mathrm{T})$ avec le débit d'exploitation (Q), puis du niveau statique (NS) avec le débit spécifique( $\left.\mathrm{Q}_{\mathrm{sp}}\right)$.

La forte corrélation entre certains paramètres a constitué le gage de la mise en relation prédictive sous la forme d'une équation mathématique à une seule 
inconnue. Le nuage formé par T et Qsp indique que ces paramètres peuvent être ajustés suivant une équation de régression de type puissance avec un coefficient de corrélation égale à 0.63 avec un exposant caractéristique égal à 1.3 (figure 5). La relation est donnée par l'équation 5 :

$$
T=0.63 Q_{s p}{ }^{1.3}
$$

$\mathrm{T}$ et $\mathrm{Q}_{\text {sp sont }}$ exprimés dans la même unité $\left(\mathrm{m}^{2} / \mathrm{h}\right)$.

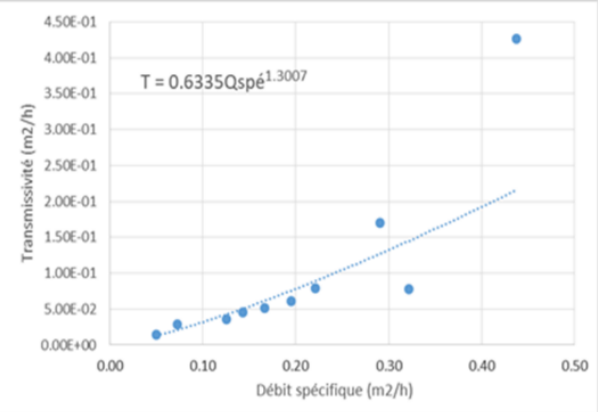

Figure 5. Droite de la relation entre la Transmissivité et le débit spécifique

De même, il est possible d'ajuster une droite de régression linéaire au nuage de points formé par le débit critique et le débit en exploitation. La relation prédictive dans un intervalle de confiance de $95 \%$ est celle d'une équation de fonction affine ( equation 6) présentée dans la figure 6.

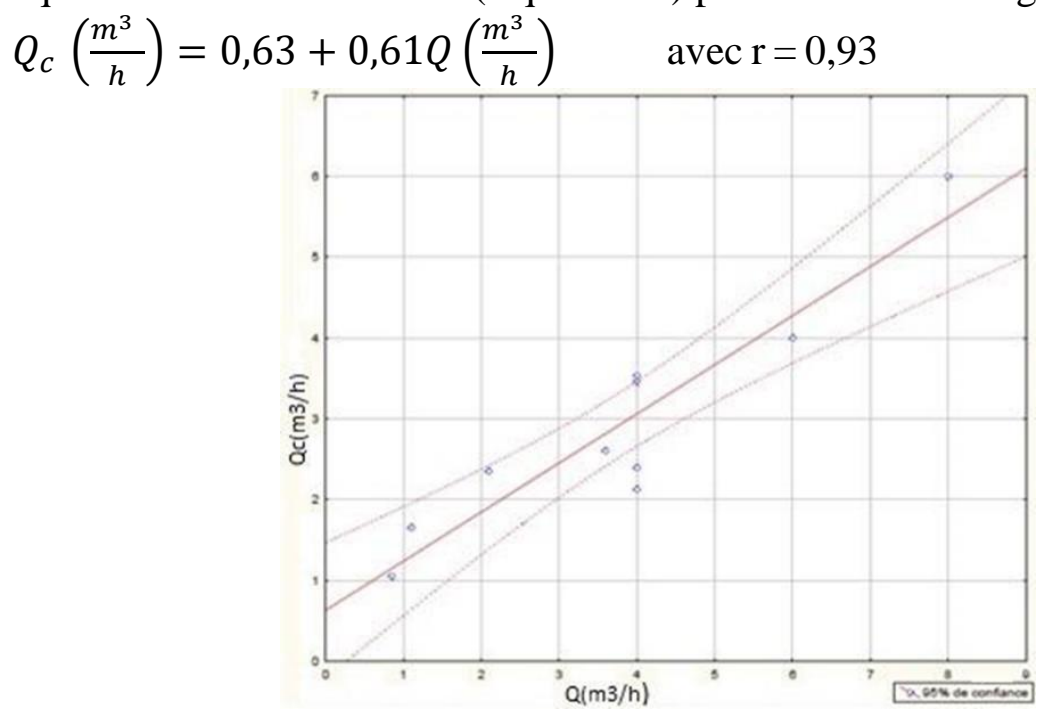

eq.(6)

Figure 6. Graphique de la relation prédictive entre le débit critique $\left(\mathrm{Q}_{c}\right)$ et le débit d'exploitation (Q).

Facteurs influençant la productivité

\begin{tabular}{|l|l|l|l|l|}
\hline Facteurs & $\begin{array}{l}\text { Valeur } \\
\text { Propre }\end{array}$ & $\begin{array}{l}\text { \% Total } \\
\text { variance }\end{array}$ & $\begin{array}{l}\text { Cumul } \\
\text { valeurs propres }\end{array}$ & \% Cumulé \\
\hline $\mathbf{1}$ & 2,96 & 49,26 & 2,96 & 49,26 \\
\hline $\mathbf{2}$ & 2,11 & 35,12 & 5,06 & 84,38 \\
\hline
\end{tabular}


Tableau IV. Valeurs propres, variances exprimées et cumulées des facteurs.

Les résultats de l'analyse multivariée ont permis d'identifier les principaux facteurs qui influencent le fonctionnement hydrogéologique des aquifères étudiés. Ces résultats sont résumés dans le tableau IV.

Les principaux facteurs sont au nombre 2, et ils représentent $84,38 \%$ de la variance totale exprimée. Les coordonnées factorielles (tableau V) ont révélé l'identité des paramètres significatifs qui contribuent à la mise en œuvre d'un facteur. Le $1^{\text {er }}$ facteur (F1) est la calibration fixée par le débit d'exploitation $(\mathrm{Q})$ et le débit critique $\left(\mathrm{Q}_{\mathrm{c}}\right)$ de l'ouvrage hydraulique, tandis que le second facteur (F2) est la potentialité hydrodynamique intrinsèque de l'aquifère à travers le débit spécifique (Qsp) et la transmissivité (T).

Tableau V. Coordonnées factorielles des variables

\begin{tabular}{|l|l|l|}
\hline \multicolumn{1}{|c|}{ Variables } & Facteur 1(F1) & Facteur 2 (F2) \\
\hline Pt & $\mathbf{0 , 8 1}$ & $-0,37$ \\
\hline $\mathbf{N S}$ & $\mathbf{- 0 , 6 6}$ & 0,41 \\
\hline $\mathbf{Q}$ & $\mathbf{0 , 9 3}$ & 0,27 \\
\hline $\mathbf{Q}_{\mathbf{c}}$ & $\mathbf{0 , 9 3}$ & 0,02 \\
\hline $\mathbf{Q}_{\text {sp }}$ & 0,37 & $\mathbf{0 , 9 2}$ \\
\hline $\mathbf{T}$ & $-0,03$ & $\mathbf{0 , 9 4}$ \\
\hline
\end{tabular}

Les paramètres qui suscitent le $1^{\text {er }}$ facteur $(\mathrm{F} 1)$ sont intelligemment fixés lors de l'équipement d'un ouvrage hydraulique dans un aquifère. Il ressort donc que le $1^{\text {er }}$ facteur de la productivité est celui des caractéristiques de l'équipement de l'ouvrage hydraulique. Le second facteur influençant la productivité est la potentialité hydrodynamique souterraine. Le cercle de communauté ou espace des variables est illustré à la Figure 7.

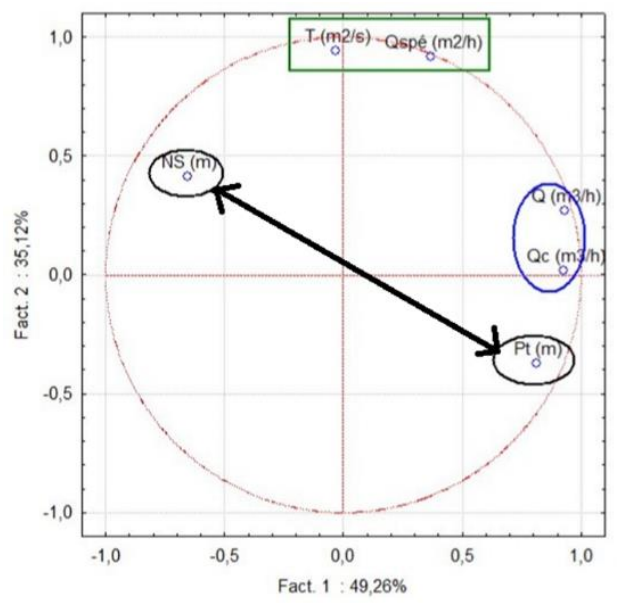

Figure 7/ Communauté des variables dans le plan factoriel F1-F2 
Cette figure montre la proximité des paramètres fortement corrélés tels que $\mathrm{T}$ et $\mathrm{Q}_{\mathrm{sp}}$, puis $\mathrm{Q}$ et $\mathrm{Q}_{\mathrm{c}}$. Il faut y noter aussi la position diamétralement opposée de Pt et NS, ce qui témoigne de l'évolution indépendante ou encore l'inexistence de relation possible entre ces deux paramètres physiques (niveau de l'eau au repos versus profondeur de forage). En définitive, la productivité des aquifères et l'efficacité des ouvrages hydrauliques dépendent respectivement des potentialités hydrodynamiques $\left(T, Q_{s p}\right)$ des aquifères et le calibrage $\left(\mathrm{Q}_{\mathrm{c}}, \mathrm{Q}\right)$ défini lors de l'équipement de l'ouvrage hydraulique avant sa mise en exploitation.

\section{Discussion}

\section{Paramètres de productivité des aquifères et ouvrages hydrauliques}

Les niveaux statiques varient entre 1,30 et $18,15 \mathrm{~m}$. La moyenne est équivalente à $7,14 \mathrm{~m}$. Les niveaux statiques sont tous inférieurs à $20 \mathrm{~m}$, ce qui est quasiment similaire à ceux observés par Ehoussou et al., (2018) dans la région du Bélier, au centre de la Côte d'Ivoire, où les niveaux statiques fréquents sont compris entre 5 et $20 \mathrm{~m}$ de profondeur. Cette similitude peut s'expliquer par le caractère similaire du contexte structural géologique du nord et du centre de la Côte d'Ivoire qui partagent la même histoire géologique et les mêmes formations géologiques (Yacé,2002).

Les débits d'exploitation des forages varient de $0,85 \mathrm{~m} 3 / \mathrm{h}$ à $8 \mathrm{~m} 3 / \mathrm{h}$ avec une moyenne de $3,77 \mathrm{~m}^{3} / \mathrm{h}$. La classification de ces débits révèle que $90 \%$ sont supérieurs à $1 \mathrm{~m}^{3} / \mathrm{h}$. Ces observations sont en accord avec celles effectuées par plusieurs auteurs en zone de socle en Côte d'Ivoire (Kouassi et al.,2012 ; Koudou et al., 2016; Ewodo et al., 2017), qui indiquent qu'un débit de $1 \mathrm{~m}^{3} / \mathrm{h}$ est suffisant dans l'approvisionnement en eau potable des zones rurales.

Les débits critiques varient entre 1,05 et $6 \mathrm{~m}^{3} / \mathrm{h}$ avec une moyenne de 2,917 $\mathrm{m}^{3} / \mathrm{h}$. Ces débits critiques sont les débits à ne pas dépasser au cours de l'exploitation des forages pour éviter la détérioration de l'ouvrage. Le débit critique est le débit qui conditionne très souvent l'exploitation d'un ouvrage hydraulique du fait qu'il permet une utilisation rationnelle de ce dernier. Ce débit permet de garantir une durabilité de la pompe à installer et surtout d'éviter l'épuisement de l'aquifère sollicité. La plupart des travaux antérieurs ne font pas cas de la valeur du débit critique ni sa relation avec le débit d'exploitation. Ce travail a le mérite de mettre en évidence ce paramètre qui permet d'apprécier le niveau de précaution établi lors de la mise en exploitation de l'ouvrage hydraulique.

Les débits spécifiques calculés varient entre $5,04.10^{-2}$ et $4,38.10^{-1} \mathrm{~m}^{2} / \mathrm{h}$ avec une moyenne de $2,02 \cdot 10^{-1} \mathrm{~m}^{2} / \mathrm{h}$. Ces débits spécifiques sont majoritairement dans la classe moyenne. Les débits spécifiques calculés ailleurs dans les zones de socle voisines sont aussi classés faibles à moyen ; notamment à Korhogo (Onetié et al., 2010) et à Katiola (Gnamba et al., 2014). 
Les transmissivités obtenues s'inscrivent dans l'intervalle de 4,01.10-6 $\mathrm{m}^{2} / \mathrm{s}$ à $1,18.10^{-4} \mathrm{~m}^{2} / \mathrm{s}$ soit une moyenne de $2,75.10^{-5} \mathrm{~m}^{2} / \mathrm{s}$. Ces valeurs observées sont dans le même ordre de grandeur que les valeurs de transmissivités brutes observées par Onetie et al. (2010) à Korhogo, Lasm et al. (2012) à Ferkéssedougou, et Assemian et al. (2014) à Bongouanou.

\section{Relation entre les paramètres hydrodynamiques}

La relation entre le débit spécifique et la transmissivité a fait l'objet d'études antérieures (Onetié et al. 2010, Lasm et al., 2012) au terme desquelles les auteurs ont établi des équations mathématiques de corrélation de ces paramètres. La très forte corrélation de 0.85 obtenue dans cette étude corrobore donc les précédents travaux. Selon Onetie et al., (2010), la valeur du coefficient de corrélation dépendrait de la solution analytique ou empirique choisie pour calculer la valeur de la transmissivité. En effet, en vue de réduire les incertitudes de résultats des solutions analytiques, Dewandel et al. (2011) recommandent vivement l'application préalable de la méthode de diagnostic qui fait l'unanimité dans l'industrie d'exploration pétrolière depuis les travaux de Bourdet et al. (1983).

\section{Conclusion}

Cette étude a permis d'évaluer les propriétés hydrodynamiques des aquifères et de déterminer le débit critique de productivité des ouvrages hydrauliques en milieu de socle. Les relations entre les différents paramètres hydrodynamiques et hydrauliques ont contribué à une meilleure identification des facteurs influençant l'exploitation de la ressource souterraine. Il s'agit de l'hydrodynamique souterraine et le calibrage des ouvrages hydrauliques avant la mise en exploitation. La présente étude a mis en évidence le niveau faible à moyen de la transmissivité et le rendement spécifique fortement corrélés puis, les équations mathématiques prédictives suivantes :

- $Q_{c}=0,63+0,61 Q$

- $T=0,63 Q_{s p} 1,30$

Bien que les débits délivrés par les aquifères et ouvrages hydrauliques réalisés sont acceptables pour une alimentation eau potable de la population en milieu rural (Hydraulique Villageoise (HV), les résultats de cette étude ont révélé les caractéristiques hydrogéologiques permettant une meilleure exploitation des réservoirs souterrains et, aussi une bonne gestion de la ressource en eau souterraine vitale. Il est donc envisagé une modélisation de l'écoulement des eaux souterraines, comme un outil d'aide à la décision au service du développement durable dans la zone étudiée et les autres régions similaires. 


\section{References:}

1. Ahoussi K. E. (2008). Évaluation quantitative et qualitative des ressources en eau dans le Sud de la Côte d'Ivoire. Application de l'hydrochimie et des isotopes de l'environnement à l'étude des aquifères continus et discontinus de la région d'Abidjan-Agboville. Thèse de Doctorat, Université de Cocody-Abidjan, Côte d'Ivoire, 270 p.

2. Assemian E. A., Kouamé K. F., Saley M. B., Affian K., Youan Ta M., Jourda J. P. \& Biemi J. (2014). Étude de la productivité d'un aquifère de socle et approche statistique pour la détermination des tranches de profondeurs potentiellement productives : cas de la région de Bongouanou, Est de la Côte d'Ivoire, revues des sciences de l'eau, vol. 27, No 1, pp. 81-97. https://doi.org/10.7202/1021984ar.

3. Atté A. G. (2010). Mise en place d'outils d'aide à la décision pour la gestion des ressources en eau en côte d'ivoire : actualisation et extension des capacités du SIG sur le bassin versant du Bandama. Mémoire de fin de cycle, master spécialisé en gestion intégrée des ressources en eau (GIRE), Institut International de l'Ingénierie de l'Eau et de l'Environnement (2iE), $50 \mathrm{p}$.

4. Biémi J. (1992). Contribution à l'étude géologique, hydrogéologique et par télédétection des bassins versants subsahariens du socle précambrien d'Afrique de l'Ouest : hydrostructurale, hydrodynamique, hydrochimie et isotopique des aquifères discontinus de sillons et des aires granitiques de la Haute Marahoué (Côte d'Ivoire), Thèse de Doctorat d'État, Université de Cocody-Abidjan, Côte d'Ivoire, $480 \mathrm{p}$.

5. Bourdet, Whittle T.M., Dougals, A.A., Pirard V.M., (1983) : A new set of type curves simplifies well test analysis, World Oil, 196, 95-106.

6. Brgm-Aquater (1991). Exploitation des eaux souterraines en socle cristallin et valorisation agricole : projet pilote expérimental en milieu rural pour des zones soudano- sahéliennes et sahéliennes. Rapport BRGM- AQUATER R 33576 Octobre 1991. BRGM, Orléans, Vol. 4.

7. C.I.E.H.(1982). Méthodes d'études et de recherches de l'eau souterraine des roches cristallines de l'Afrique de l'ouest, Atlas de photo-interprétation, Vol. 3, $143 \mathrm{p}$.

8. Cooper H. H. \& Jacob C. C. (1946). A generalized graphiquical method for evaluating formation constants and summarizing well field history. Am. Geophys. UnionTrans., Vol. 27, No. 04, pp. 526-534.

9. De Lasme O. Z. (2013). Contribution à une meilleure connaissance des aquifères fissures du socle précambrien: cas de la région de San Pedro (Sud-Ouest de la Côte d'Ivoire). Thèse de doctorat Hydrogéologie, Université Félix Houphouët Boigny, Cocody, 176 p. 
10. De Lasme O. Z., Lasm T., Oga M.-S., Youan T. M., Baka D., Ettien F. B., Kouakou S., Onetie O. Z, \& Yao T. K. (2012). Analyse des propriétés hydrodynamiques des aquifères fissurés de la région de SanPedro (Sud-Ouest de la Côte d'Ivoire), Estudios Geológicos, No 68, pp 217-232.

11. Dewandel B., Lachassagne, P., Zaidi, F.K., Chandra, S., (2011) : A conceptual hydrodynamic model of a geological discontinuity in hard rock aquifers: example of a quartz reef in granitic terrain in South India. Journal of Hydrology, 405, 474-487.

12. Duffield, G.M., (2007). AQTESOLV for Windows Version 4.5 User's Guide, HydroSOLVE, Inc., Reston, VA

13. Ehoussou K.M., Kouassi A.M., Kamagate B. (2018). Caractérisation hydrodynamique des aquifères fissurés de la région du bélier (centre de la côte d'ivoire), Larhyss Journal, No 36, pp. 119-143

14. Ewodo M. G., Bon A. F., Bineli E., Ntep F. \& Ombolo A. (2017). Caractérisation de la productivité des aquifères du socle de la région de l'extrême nord, Cameroun, journal of the cameroon academy of sciences, Vol. 14, No 1, pp. 44-60.

15. Faillat J.P. (1987). Aquifères fissurés en zone tropicale humide: structure, hydrodynamique et hydrochimie (Afrique de l'ouest). Hydrogéologie, Thèse Université, Languedoc (Montpellier), 534 p.

16. Geomines (1982). Inventaire hydrogéologique appliqué à l'hydraulique villageoise. Carte de Niellé, cahier n³, (1982a), $20 \mathrm{p}$.

17. Gnamba F.M., Oga Y.M.S., Gnangne, T. \& Lasm T., (2014). Analyse de la productivité des aquifères de fissures du socle Paléoprotérozoïque de la région de Katiola (Centre-Nord de la Côte d'Ivoire). European Scientific Journal, Vol 10, No 5, pp. 79-98.

18. Gutierrez A., Dewandel B. (2013). L'interprétation des pompages d'essais : quelles nouveautés? Géologues n 178 . Octobre 2013.pp 4953

19. Jourda J. P. (2005). Méthodologie d'application des techniques de télédétection et des systèmes d'information géographique à l'étude des aquifères fissurés d'Afrique de l'ouest. Concept de l'Hydrotechnique spatiale: cas des zones tests de la Côte d'Ivoire", Thèse de doctorat ès Sciences Naturelles, Université de Cocody-Abidjan, Côte d'Ivoire, $429 \mathrm{p}$.

20. Koita M. (2010). Caractérisation Et Modélisation Du Fonctionnement Hydrodynamique D'un Aquifère Fracturé En Zone De Socle. Région De Dimbokro-Bongouanou (Centre Est De La Côte d'Ivoire). Thèse de doctorat, Université de Montpelier II, 235 p.

21. Konan A.S. ( 2018) 
http://www.jeuneafrique.com/559212/politique/cote-divoire-legouvernement-face-a-une-vaste-penurie-deau-courante-dans-le-norddu-pays/

22. Kouakou K. E. G. (2012). Contribution de la géophysique à l'étude structurale et à la connaissance des ressources en eau souterraine du district de la vallée du Bandama (Côte d'Ivoire), Thèse de doctorat géophysique appliquée, Université Félix Houphouët Boigny, Cocody, $199 \mathrm{p}$.

23. Kouakou K. E. G., Sombo B. C., Digbéhi Z. B., Kouassi F. W., Sombo P., \& Kouamé N. (2012). Utilisation de la prospection géophysique par résistivité électrique pour la recherche d'eau souterraine dans le département de TANDA (Est de la Côte d'Ivoire). European Journal of Scientific Research, Vol. 83, No 3, pp. 230-244.

24. Kouassi K. X. (2011). Évaluation quantitative et qualitative des ressources en eau de la région centre: cas du département de Didiévi en Côte d'Ivoire. Mémoire de fin de cycle Ingénieur de conception en exploitation et traitement des eaux, Institut national polytechnique Félix Houphouët-Boigny de Yamoussoukro (Côte d'Ivoire). $50 \mathrm{p}$

25. Koudou A., N'dri B. E., Niamke K. H., Adjiri O. A., Sombo A. P. \& Niangoran K. C. (2016). Analyse de la productivité et estimation de la vulnérabilité à la pollution pour l'optimisation de l'implantation des forages dans les aquifères de socle du bassin versant du N'zi (Côte d'Ivoire). Bulletin de l'Institut Scientifique, Rabat, Section Sciences de la Terre, No 38, pp. 149-164.

26. Lachassagne P., Dewandel B., Wyns R. (2014). The conceptual model of weathered hard rock aquifers and its practical applications. Chapter in Book Fractured rock Hydrogeology. International Association of Hydrogreologist selected papers. Editor : John M. Sharp.

27. Lasm T, Fossou R, N'guessan M, Onetie Oz, Baka D, Youan T. M, Oga M. S, \&"Soro N. (2012). Contribution hydrogéologique à la connaissance des aquifères discontinus du département de Ferké (nord de la Côte d'Ivoire) pour une meilleure alimentation en eau potable. Revue Ivoirienne des Sciences et Technologie, No 19, pp.114-135.

28. Lasm T. (2000). Hydrogéologie des réservoirs fracturés de socle : Analyse statistique de la fracturation et des propriétés hydrodynamiques. Application à la région des montagnes de Côte d'Ivoire (domaine archéen), Thèse de doctorat, Université, Poitiers, France, $274 \mathrm{p}$.

29. N'go Y. A. Gone D. L, Savane I. \& Goble M. M. (2005). Potentialités en eaux souterraines des aquifères fissurés de la région d'Agboville (Sud de la Côte d'Ivoire) : caractérisation hydroclimatique et physique. Afrique Science, Vol 1, No 1, pp. 127-144. 
30. Onetie O. Z., Lasm T., Baka D., N., M. R. Fossou, S. O. Kouakou, Youan Ta. M., De Lasme O. Z., Oga M. S. \& Soro N. (2010). Influence des pertes de charges quadratiques sur la transmissivité et le débit spécifique déterminés sur des forages isolés : cas de la région de Korhogo au nord de la côte d'ivoire, Revue Ivoirienne des Sciences et Technologie, No 16, pp. 261-283.

31. Soro G. (2010). Évaluation quantitative et qualitative des ressources en eau souterraines dans la région des Lacs (centre de la Côte d'Ivoire) : Hydrogéologie et Hydrochimie des aquifères discontinus du district de Yamoussoukro et du département de Tiébissou, Thèse de doctorat, Université de Cocody, Abidjan, Côte d'Ivoire, $257 \mathrm{p}$.

32. Soro G. Soro N. Ahoussi K. E. Lasm T. Kouame F. K.; Soro T. D.Et Biemi J. (2010). Évaluation des propriétés hydrauliques des aquifères fracturés des formations cristalline et métamorphique dans la région des Lacs (centre de la Côte d'Ivoire). Estudios Geológicos, Vol 66, No 2, pp 227-242.

33. Yacé I. (2002). Initiation à la Géologie. L'exemple de la Côte d"Ivoire et de L'Afrique de 1'Ouest. Édition Ceda, 183 p. 